\title{
Non-parametric methods for recurrent event data with informative and non-informative censorings
}

\author{
Mei-Cheng Wang ${ }^{1, *, \dagger}$ and Chin-Tsang Chiang ${ }^{2}$ \\ ${ }^{1}$ Department of Biostatistics, Johns Hopkins University, Baltimore, U.S.A. \\ ${ }^{2}$ Department of Mathematics, National Taiwan University, Taiwan
}

\begin{abstract}
SUMMARY
Recurrent event data are commonly encountered in health-related longitudinal studies. In this paper timeto-events models for recurrent event data are studied with non-informative and informative censorings. In statistical literature, the risk set methods have been confirmed to serve as an appropriate and efficient approach for analysing recurrent event data when censoring is non-informative. This approach produces biased results, however, when censoring is informative for the time-to-events outcome data. We compare the risk set methods with alternative non-parametric approaches which are robust subject to informative censoring. In particular, non-parametric procedures for the estimation of the cumulative occurrence rate function (CORF) and the occurrence rate function (ORF) are discussed in detail. Simulation and an analysis of data from the AIDS Link to Intravenous Experiences Cohort Study is presented. Copyright (C) 2002 John Wiley \& Sons, Ltd.
\end{abstract}

KEY WORDS: cumulative rate function; informative censoring; intensity function; kernal estimation; rate function; recurrent events

\section{INTRODUCTION}

Recurrent event data can be regarded as a specific type of correlated data with the feature that event times of a subject are ordered and correlated. Such data are frequently encounted in health-related studies where longitudinal follow-up designs are commonly employed. Using the Intravenous Experiences Cohort Study as an example, drug users may be repeatedly hospitalized due to drug-associated disease symptoms; the typical health service data consist of information from multiple outpatient and inpatient treatment, ambulance uses, and emergency room visits [1]. Other examples include multiple live births in a woman's lifetime, repeated breakdowns of an automobile, multiple opportunistic infections in studies of acquired immunodeficiency syndrome (AIDS), and multiple injuries in ageing studies.

\footnotetext{
*Correspondence to: Mei-Cheng Wang, Department of Biostatistics, Johns Hopkins University, 615 N. Wolfe St., Baltimore, MD 21205, U.S.A.

†E-mail: mcwang@jhsph.edu
}

Contract/grant sponsor: NIDA; contract/grant numbers: DA04334, DA08009

Contract/grant sponsor: National Institute of Health; contract/grant numbers: R01 HD38209, R01 MH56639

Copyright (c) 2002 John Wiley \& Sons, Ltd.

Accepted April 2001 
In this paper, we study recurrent events of the same type. To analyse recurrent event data, the focus could be placed on time-between-events or time-to-events analysis. When time-between-events is the variable of interest, statistical approaches were proposed for nonparametric estimation of the recurrent survival function [2], and for regression analysis [3]. The focus of this paper is on time-to-events data and we consider modelling the occurrence rate of recurrent events in a specified time interval $\left[0, T_{0}\right]$. Here 0 refers to the time origin which could be either a calendar time or a medically-defined time such as the disease onset or treatment onset. The constant $T_{0}>0$ is determined with the knowledge that recurrent events could potentially be observed at least up to $T_{0}$. Practically, $T_{0}$ is chosen to be a point of time which is less than the maximum of the observed recurrent event times. Let $N(t)$ represent the number of recurrent events occurring prior to or at $t, t \in\left[0, T_{0}\right]$. The occurrence rate function (ORF) of a continuous recurrent event process at $t, t \in\left[0, T_{0}\right]$, is defined as

$$
\lambda(t)=\lim _{\Delta \rightarrow 0^{+}} \frac{\operatorname{Pr}(N(t+\Delta)-N(t)>0)}{\Delta}
$$

Note that the ORF is quantitatively different from the conventional intensity function of a point process where the intensity is defined as the occurrence rate of recurrent events conditional on the event history up to $t$. In contrast with the conditional interpretation of the intensity function, the ORF is defined as the population average of occurrence rate of recurrent events at time point $t$ unconditionally on the event history. Conceptually, in many biomedical applications, the ORF is preferred over the intensity function because it gives more direct interpretations for identifying treatment effects and for comparison of risk factors. In general, use of an intensity model appears more appropriate for individual-based prediction and use of the ORF is more relevant for population-based comparison. The cumulative occurrence rate function (CORF) is defined as

$$
\Lambda(t)=\int_{0}^{t} \lambda(u) \mathrm{d} u, \quad t \in\left[0, T_{0}\right]
$$

The CORF $\Lambda(t)$ is also the expectation of the number of recurrent events occurring in $[0, t]$.

In this paper, the research interest is focused on the occurrence rate of recurrent events. We study and compare non-parametric procedures for the estimation of the CORF and ORF under non-informative and informative censoring models. The non-parametric methods are applied to the analysis of data from the AIDS Link to Intravenous Experiences Cohort Study.

\section{NON-INFORMATIVE CENSORING MODEL: RISK-SET METHODS}

For subject $i, i=1,2, \ldots, n$, let $N_{i}(t)$ denote the number of recurrent events occurring at or prior to $t, t \geqslant 0$, and $y_{i}$ the censoring time at which the observation of the recurrent events is terminated. Let $t_{i 1} \leqslant \cdots \leqslant t_{i, m_{i}}$ be the ordered event times with $m_{i}$ defined as the index for the last event occurring at or prior to $y_{i}$.

\subsection{CORF estimation}

In univariate survival analysis, the concept of 'risk sets' plays a key role for the development of many non-parametric and semi-parametric methodologies. For a single event process (that is, univariate survival analysis), the risk population defined at $t$ comprises the subjects who 
have not experienced the failure event, and a risk set at $t$ consists of the subjects whose observed failure time, censored or uncensored, is not less than $t$. It is well known that this risk set forms a random sample from the corresponding risk population when censoring is non-informative.

For the recurrent event process, the risk set at $t$ is defined as $R(t)=\left\{i: y_{i} \geqslant t\right\}$ which includes subjects who are under observation at $t$. When the censoring time $Y_{i}$ is independent of the recurrent event process $N_{i}(\cdot)$, the risk set forms a random sample from the risk population at $t$. It is important to indicate that the risk population of a single event process is composed of subjects who have not failed prior to $t$, thus the risk population varies with different values of $t$. In contrast, for a recurrent event process, the risk population at different $t \mathrm{~s}$ always coincides with the target population defined at 0 .

Let $n_{R}(t)$ be the number of subjects in the risk set $R(t)$. Under the non-informative censoring assumption, for $t>0$ and positive-valued but small $\Delta$, a crude estimate of the occurrence probability in $(t-\Delta, t]$ can be constructed as

$$
\lambda(t) \Delta \approx \frac{\sum_{i=1}^{n} \sum_{j=1}^{m_{i}} I\left(t_{i j} \in(t-\Delta, t]\right)}{n_{R}(t)}
$$

with $I(\cdot)$ representing the indicator function. The estimate is essentially an empirical measure with time-dependent sample size $n_{R}(t)$. A non-parametric estimate of the CORF corresponding to (1) can then be constructed as

$$
\hat{\Lambda}(t)=\sum_{i=1}^{n} \sum_{j=1}^{m_{i}} \frac{I\left(t_{i j} \leqslant t\right)}{n_{R}\left(t_{i j}\right)}
$$

This estimator together with its properties have been studied in the literature by Nelson [4], Lawless and Nadeau [5], and Pepe and Cai [6]. Further estimators which incorporate with covariate information into the estimation were developed by Cook and Lawless [7].

More generally, a functional transformation of the CORF, $g(\Lambda)$, can be estimated by $g(\hat{\Lambda})$. For instance, define $g(\Lambda)=\Lambda^{-1}(c)$ where $c>0$ is a constant, and $\Lambda^{-1}(c)$ can be estimated by $\hat{\Lambda}^{-1}(c)$. In the ALIVE study, this estimator can be interpreted as the time length in which drug users are expected to be hospitalized $c$ times. Also, non-parametric estimators of the expected number of events occurring in the intervals $\left(0, b_{1}\right],\left(b_{1}, b_{2}\right], \ldots,\left(b_{k-1}, b_{k}\right]$ can be constructed as $\left(\hat{\Lambda}\left(b_{1}\right)-\hat{\Lambda}(0), \hat{\Lambda}\left(b_{2}\right)-\hat{\Lambda}\left(b_{1}\right), \ldots, \hat{\Lambda}\left(b_{k}\right)-\hat{\Lambda}\left(b_{k-1}\right)\right)$.

\subsection{ORF estimation}

In addition to the non-informative censoring assumption, further assume that, for the $i$ th subject, $N_{i}(t)$ is distributed as a non-stationary Poisson process with the subject-specific intensity function $\lambda_{i}(t)$. Here the average of $\lambda_{i}(t), \lambda(t)=E\left[\lambda_{i}(t)\right]$, is the ORF of recurrent events for the target population. The estimation of the ORF requires use of smoothing techniques. We first consider the kernel estimator of the subject-specific intensity function $\lambda_{i}(t)$ for $t$ in the interval $\left[0, y_{i}\right]$ by

$$
\hat{\lambda}_{i}(t)=\sum_{j=1}^{m_{i}} K_{y_{i}}\left(\frac{t-t_{i j}}{h}\right)
$$

where $K_{y_{i}}(\cdot)$ is a boundary kernel density of Gasser and Müller [8] with adjustment for the censoring time $y_{i}$, and $h$ is a positive-valued bandwidth. By averaging the subject-specific 
estimators of subjects who are still at risk at $t$, the kernel estimator, say, $\hat{\lambda}_{h}(t)$, for $\lambda(t)$ is then given by

$$
\hat{\lambda}_{h}(t)=\sum_{i=1}^{n}\left(\frac{I\left(y_{i} \geqslant t\right)}{n_{R}(t)}\right) \hat{\lambda}_{i}(t), \quad t \in\left[0, T_{0}\right]
$$

Because the censoring is independent of the recurrent event process, the risk set at each $t$ forms a random sample from the population and the smoothing technique uses the risk set as the base for kernel estimation.

Other non-parametric techniques established in the literature include those in Pepe and Cai [6] and Lawless et al. [9]. In addition, when the censoring times coincide with a pre-fixed constant (that is, $y_{i}=T_{0}$ ), some non-parametric smoothing methods were also proposed [10].

\section{INFORMATIVE CENSORING MODEL}

To analyse recurrent event data, the non-informative censoring assumption is usually required for the validity of statistical methods. In many applications, however, censoring could be caused by informative drop-out or death, and it is unrealistic to assume the independence censoring condition. As noted in the previous section, the risk set techniques serve as useful tools for non-parametric estimation in non-informative censoring models. When censoring is informative about the event process, the risk set methodology breaks down because subjects in the risk set do not form a representative sample from the target population. In this section we consider a multiplicative intensity model in which the dependence of censoring and recurrent event process is explained by a latent variable. The model consists of the following assumptions:

(a) $N_{i}(t)$ is a non-stationary Poisson process with the subject-specific intensity function $\lambda_{i}(t)=z_{i} \lambda_{0}(t)$, where the baseline intensity $\lambda_{0}(t)$ is a continuous function and $Z_{i}$ is a non-negative latent variable with the distribution $H$.

(b) Conditioning on $z_{i}, N_{i}(\cdot)$ is independent of $Y_{i}$.

The latent variable $Z_{i}$ in (a) can also be viewed as the frailty or random effect which characterizes subject heterogeneity in the population. The recurrent event process in (a) is a multiplicative intensity model in which the baseline intensity function remains the same for each individual, but the magnitude of the intensity varies according to the latent variable value. Assumption (b) assumes the censoring time is independent of the recurrent event process given the value of the latent variable. Under (b), the distribution of the censoring time, $Y_{i}$, is allowed to depend on $z_{i}$ and this substantially relaxes the usual non-informative censoring constraint. As a main feature of our method procedures, which will be presented below, the distributions of the censoring and latent variables are both treated as nuisance functions, and we avoid modelling and estimating the nuisance functions by proper procedures.

Under the multiplicative intensity model (a), note that due to the independent increment property of Poisson processes, the intensity function is the same as the ORF given $z_{i}$. The ORF is $\lambda(t)=\mu_{z} \lambda_{0}(t)$ and the CORF is $\Lambda(t)=\int_{0}^{t} \lambda(u) \mathrm{d} u=\mu_{z} \Lambda_{0}(t)$, with $\mu_{z}=E\left[Z_{i}\right]$. 


\subsection{CORF estimation}

Define the 'shape function' as

$$
f(t)=\frac{\lambda_{0}(t)}{\Lambda_{0}\left(T_{0}\right)}=\frac{z_{i} \lambda_{0}(t)}{z_{i} \Lambda_{0}\left(T_{0}\right)}=\frac{\lambda_{i}(t)}{\int_{0}^{T_{0}} \lambda_{i}(u) \mathrm{d} u}, \quad t \in\left[0, T_{0}\right]
$$

Under (a) the shape function remains invariant for subjects in the population. It is clear that $f$ is a probability density function. Denote by $F$ the cumulative distribution function of $f$. For the CORF estimation, we will first estimate the shape function and then estimate the total magnitude of occurrence rate, $\Lambda\left(T_{0}\right)$, by a projection technique. An estimator of $\Lambda(t)$ can be achieved by taking the product of the two estimators.

Conditional on $\left(z_{i}, y_{i}, m_{i}\right),\left(t_{i 1}, t_{i 2}, \ldots, t_{i, m_{i}}\right)$ are the order statistics of independent and identically distributed random variables with density $f(t) / F\left(y_{i}\right), 0 \leqslant t \leqslant y_{i}$. Thus, conditional on $\left(y_{i}, m_{i}\right)$, the likelihood function of $\left(t_{i 1}, t_{i 2}, \ldots, t_{i, m_{i}}\right)$ is

$$
L_{\mathrm{c}}=\prod_{i=1}^{n} \int\left\{m_{i} ! \prod_{j=1}^{m_{i}} \frac{f\left(t_{i j}\right)}{F\left(y_{i}\right)}\right\} \mathrm{d} H\left(z_{i}\right) \propto \prod_{i=1}^{n} \prod_{j=1}^{m_{i}} \frac{f\left(t_{i j}\right)}{F\left(y_{i}\right)}
$$

With regularity conditions, the likelihood function $L_{\mathrm{c}}$ is maximized by the truncation productlimit estimator $[11,12]$

$$
\hat{F}(t)=\prod_{s_{(l)}>t}\left(1-\frac{d_{(l)}}{N_{(l)}}\right)
$$

where $\left\{s_{(l)}\right\}$ represent the ordered and distinct values of $\left\{t_{i j}\right\}, d_{(l)}$ is the number of events occurring at $s_{(l)}$, and $N_{(l)}$ is the total number of events with event time and censoring time satisfying $t_{i j} \leqslant s_{(l)} \leqslant y_{i}$.

With $F$ estimated by $\hat{F}$, we consider in the next step the estimation of $\Lambda\left(T_{0}\right)$. Conditioning on $\left(y_{i}, z_{i}\right)$, the number of the observed events, $m_{i}$, has the Poisson distribution with the expected value $z_{i} \Lambda_{0}\left(y_{i}\right)$. The number of events in $\left[0, T_{0}\right]$, for the $i$ th subject, can therefore be projected by $m_{i} / F\left(Y_{i}\right)$. Estimate $\Lambda\left(T_{0}\right)$ by $\hat{\Lambda}\left(T_{0}\right)=n^{-1} \sum_{i} m_{i} / \hat{F}\left(Y_{i}\right)$ and we derive an estimator of $\Lambda(t)$ as $\hat{\Lambda}(t)=\hat{F}(t) \hat{\Lambda}\left(T_{0}\right)$. Large sample properties of this estimator were confirmed and studied in detail by Wang et al. [13].

\subsection{ORF estimation}

Under the informative censoring model ((a) and (b)), the estimator $\hat{\lambda}_{h}(t)$ in (3) is generally biased even when the number of subjects is large. To handle the informative censoring, we first estimate the density $f_{i}^{*}(t)=f(t) / F\left(y_{i}\right)$, based on the $i$ th subject's data, by

$$
\tilde{f}_{i}^{*}(t)=\frac{1}{m_{i}} \sum_{j=1}^{m_{i}} K_{y_{i}}\left(\frac{t-t_{i j}}{h}\right)
$$

Note that the recurrent event process $N_{i}(t)$ is truncated at $y_{i}$ and each subject can provide information only from the time interval $\left[0, y_{i}\right]$. Also, $\lambda(t)=f_{i}^{*}(t) \Lambda\left(y_{i}\right)$ for $t \in\left[0, y_{i}\right]$. 
Substituting $f_{i}^{*}(t)$ and $\Lambda\left(y_{i}\right)$ by $\tilde{f}_{i}^{*}(t)$ and $\hat{\Lambda}\left(y_{i}\right)$, a subject-specific estimator can be obtained as $\tilde{\lambda}_{i}(t)=\tilde{f}_{i}^{*}(t) \hat{\Lambda}\left(y_{i}\right)$ if the $i$ th subject is at risk at $t$. Thus, with the above bias adjustment, an estimator can be constructed as the average of those subject-specific estimators provided by the subjects who are at risk at $t$

$$
\tilde{\lambda}_{h}(t)=\sum_{i=1}^{n}\left(\frac{I\left(y_{i} \geqslant t\right)}{n_{R}(t)}\right) \tilde{\lambda}_{i}(t), \quad t \in\left[0, T_{0}\right]
$$

Mathematical proofs and technical details for the appropriateness of the kernel estimators (3) and (5) were established by Chiang and Wang (submitted manuscript).

\section{SIMULATION}

To examine the performance of the estimation methods under the validities of non-informative and informative censoring models, a Monte Carlo simulation is implemented. In the simulation, the data are repeatedly generated 500 times from 400 independent non-stationary Poisson processes $\left\{N_{i}(t)\right\}$ with the corresponding subject-specific intensity function $\lambda_{i}(t)=z_{i} \lambda_{0}(t)$, where

$$
\lambda_{0}(t)=2+\frac{(t-3)^{3}}{27}, \quad t \in[0,5]
$$

and the latent variable $z_{i}$ is generated by four different uniform distributions: (i) $\mathrm{U}(1.25,1.25)$; (ii) $\mathrm{U}(0.75,1.75)$; (iii) $\mathrm{U}(0.5,2)$; and (iv) $\mathrm{U}(0.25,2.25)$. The baseline $\mathrm{ORF} \lambda_{0}(t)$ is chosen to simulate the situation that the occurrence probability of recurrent events increases over time. The latent variable distribution is chosen to be uniformly distributed with four ranges to represent different levels of informative censoring. Note that the informative censoring is degenerated to non-informative censoring when the uniform distribution of $z_{i}$ is centred (degenerated) at a point. In case (i), the distribution of $z_{i}$ is degenerated at 1.25 and the model becomes a non-informative censoring model. We experimented with four distributions on $z_{i}$ to examine the bias generated by different degrees of informative censoring and to confirm the appropriateness of the proposed estimation procedures. When the latent variable follows the degenerated uniform distribution of case (i), the informative and non-informative censoring models coincide with each other. Conditioning on $z_{i}$, the censoring time $y_{i}$ is set to be distributed as a truncated distribution of the exponential distribution $\exp \left(z_{i}\right)$, where the truncated distribution ranges from 1 to 5 and has the density

$$
f_{Y \mid z_{i}}(y)=\frac{z_{i} \exp \left(-z_{i} y\right)}{\exp \left(-z_{i}\right)-\exp \left(-5 z_{i}\right)}, \quad y \in[1,5]
$$

The risk-set methods in Section 2 and the proposed methods in Section 3 are used to compute the estimates of the CORF and ORF. Here, the Gaussian kernel and an adequate subjective bandwidth are used in the kernel estimators. Alternative selections of adequate kernel function and bandwidth are also possible. Based on 500 generated data sets for each simulation model. Figure 1 presents the true curves as well as the averages of the estimated $\mathrm{CORF}$ and ORF for the informative and non-informative censoring models, with the latent 
$U(1.25,1.25)$

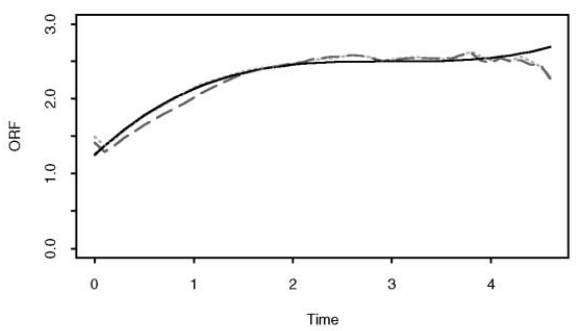

$\mathrm{U}(0.75,1.75)$

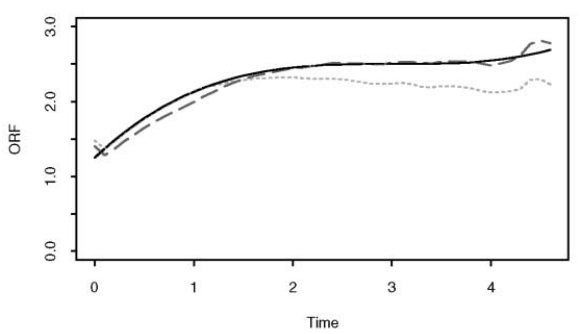

$U(0.5,2)$

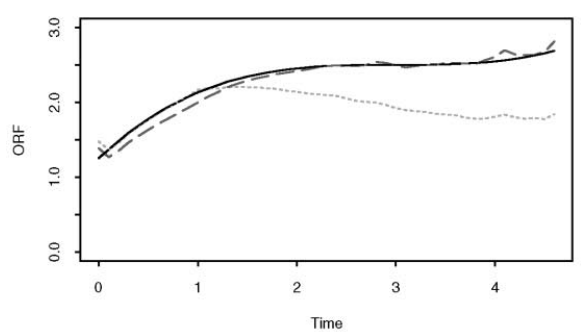

$U(0.25,2.25)$

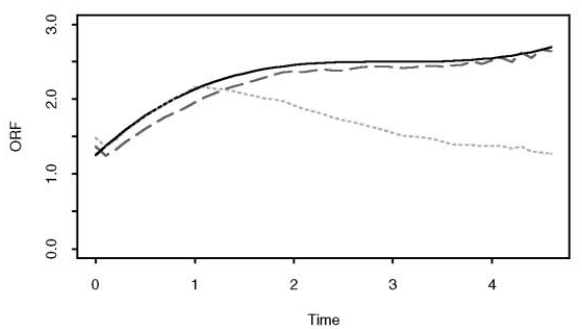

$U(1.25,1.25)$

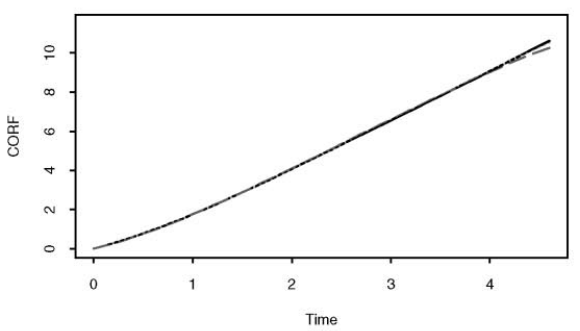

$\mathrm{U}(0.75,1.75)$

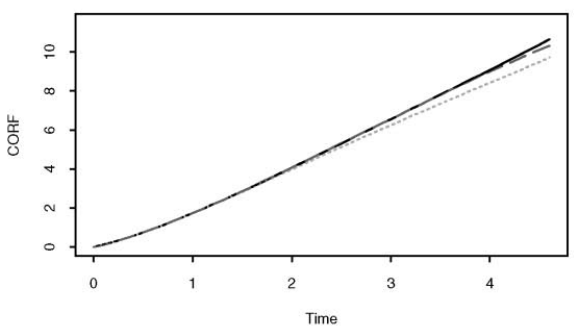

$U(0.5,2)$

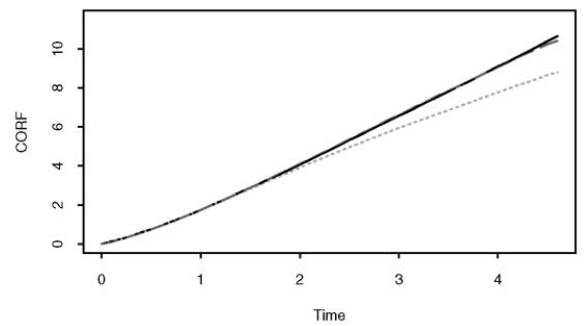

$U(0.25,2.25)$

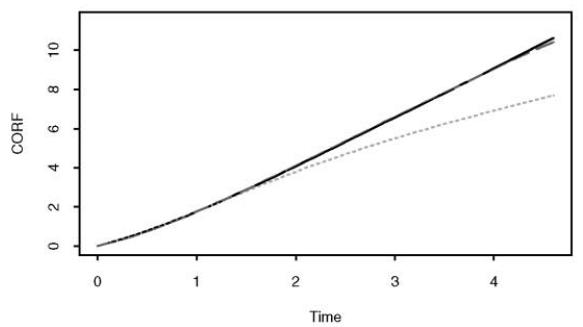

Figure 1. The real CORF and ORF (solid curve), the non-informative censoring estimated curve (dotted curve), and the informative censoring estimated curve (broken curve).

variable distribution chosen from (i)-(iv). As shown in these figures, the risk-set methods produce a more significant bias as the variance of the latent variable becomes large, whereas, the proposed methods always provide appropriate estimates. Note that the risk-set methods 
tend to be more efficient than the proposed methods when the censoring mechanism is noninformative. This is, however, a typical trade-off between efficiency and robustness of statistical methodologies which comes as no surprise.

\section{APPLICATION TO THE ALIVE STUDY}

In this section we use the data of the AIDS Link to Intravenous Experiences (ALIVE) cohort study for the illustration of the estimation methods under non-informative and informative censoring models. The study was initiated in 1988, and started systematically to collect the health service information of the intravenous drug users in July 1993. To ensure better quality of data, our data analysis uses the hospitalization data collected after 1 August 1993. Here, we only consider the repeated hospitalization records of $450 \mathrm{HIV}$-negative and $297 \mathrm{HIV}$-positive intravenous drug users, who entered the study prior to 1 August 1993 and were observed between 1 August 1993 and 31 December 1997. In the analysis the HIV status was defined as the status confirmed at the study entry, 0 (1 August 1993). A small number of HIV-negative drug users who became HIV-positive during the study period are excluded from analysis.

Let the observed dates of inpatient admission be represented by $t_{i 1} \leqslant \cdots \leqslant t_{i, m_{i}}$. The censoring time, $y_{i}$, is the time length from 1 August 1993 to the last follow-up visit of subject $i$. For HIV-negative drug users, the median of the number of recurrent events is 1 and the number ranges from 0 to 19 . The median of the censoring time is 4.12 years and the censoring time ranges from 0.275 to 4.394 years. For HIV-positive drug users, the median of the number of recurrent events is 2 and the number ranges from 0 to 14 . The median of the censoring time is 3.939 years and the censoring time ranges from 0.047 to 4.394 years. The main objectives of our analysis are to provide appropriate CORF and ORF estimators of the hospitalization rates for drug users. Meanwhile, the bootstrap confidence intervals are constructed to evaluate the accuracy of the estimated curves.

Based on the estimation methods described in Sections 2 and 3, we compute the CORF and ORF curves for HIV-negative and HIV-positive drug user groups. Similar to the simulation analysis, the Gaussian kernel and an adequate subjective bandwidth are used in the kernel estimators. The approximate 95 per cent bootstrap confidence intervals are constructed using the empirical distributions of the bootstrap analogues. As shown in Figures 2 and 3, the estimated curves for HIV-negative drug users are similar under non-informative and informative censoring models; however, for the HIV-positive group, the risk-set methods produced hospitalization rates lower than those produced by the proposed methods. We suspect that this analytical difference might be caused by informative drop-outs from sicker HIV-positive users. Moreover, from these figures, we see that HIV-positive drug users have significantly higher hospitalization rates than HIV-negative users under both approaches. Figure 2 reveals that the occurrence rates of the HIV-positive drug user group are greater than 1 after the starting short period, and the hospitalization rates of HIV-negative ones are lower than 1. An alternative way is to derive crude estimates of the expected number of events occurring every six month, and use these estimates to compare the differences between the two drug user groups. Based on the CORF and ORF estimation results, it appears appropriate to use the risk-set methods of the non-informative censoring model for HIV-negative drug users, and the proposed methods of the informative censoring model for HIV-positive drug users, respectively, to produce these crude estimates. In Tables I and II, one can see that the estimated numbers of events for 
HIV Positive Drug Users

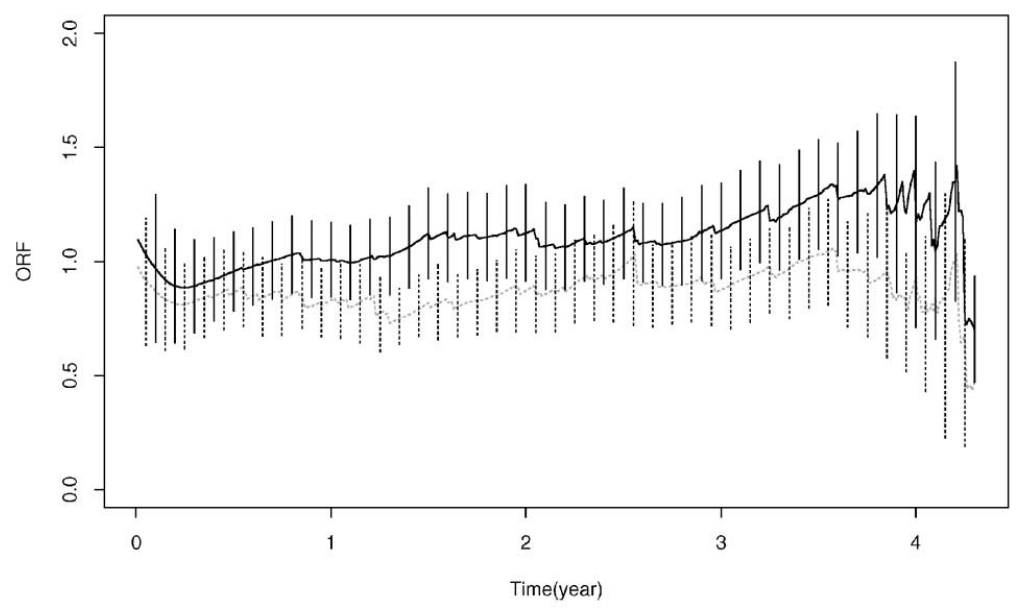

HIV Negative Drug Users

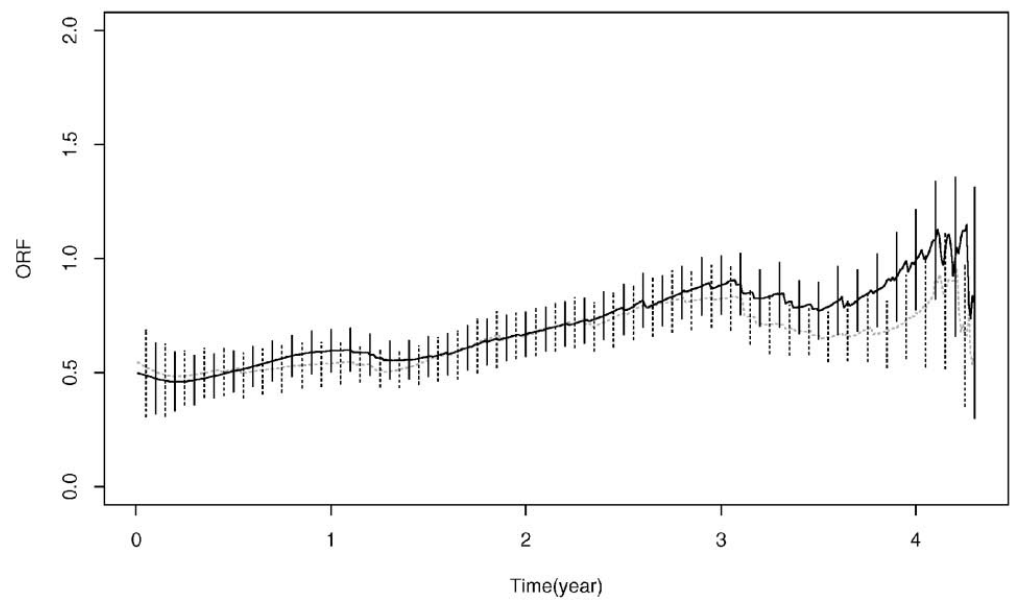

Figure 2. The estimated ORF for HIV-positive and HIV-negative drug user groups with 95 per cent bootstrap confidence intervals. Dotted curves: non-informative censoring estimation method; solid curves: informative censoring estimation method.

HIV-negative drug users are uniformly smaller than those of HIV-positive drug users. These are consistent with the conclusion of the ORF analysis.

\section{DISCUSSION}

In this paper non-parametric procedures for the estimation of the CORF and ORF in informative and non-informative censoring models are discussed. An application to the ALIVE study is presented to compare the two different types of methodologies. 


\section{HIV Positive Drug Users}

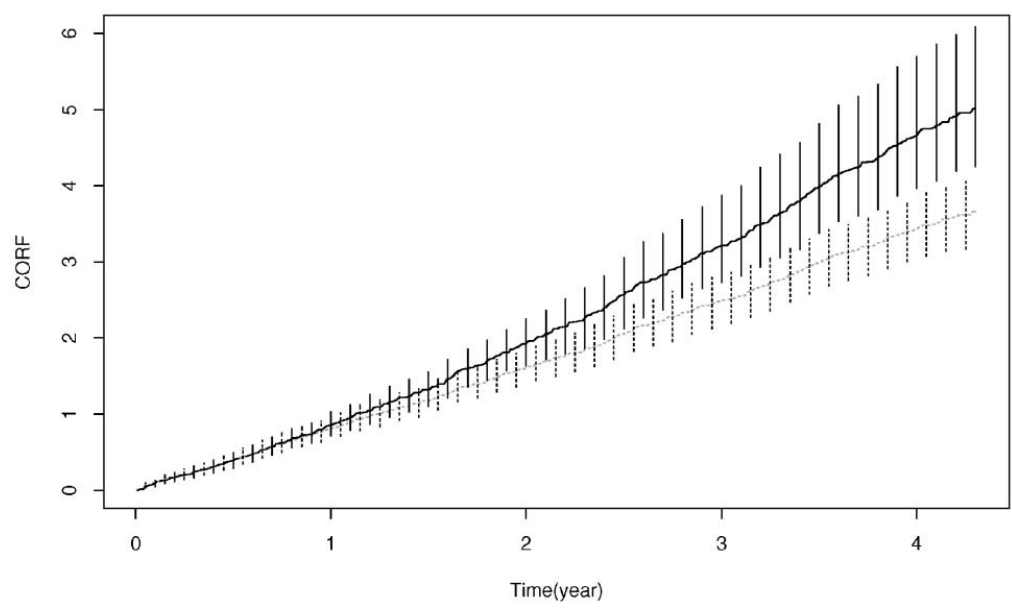

HIV Negative Drug Users

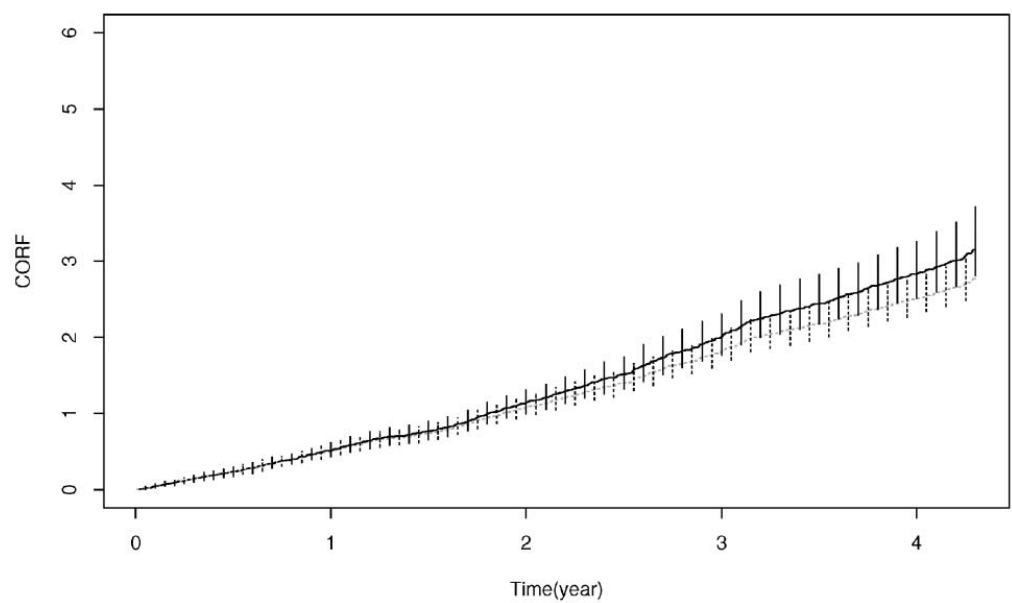

Figure 3. The estimated CORF for HIV-positive and HIV-negative drug user groups with 95 per cent bootstrap confidence intervals. Dotted curves: non-informative censoring estimation method; solid curves: informative censoring estimation method.

In the analysis of recurrent event data, parametric models and maximum likelihood techniques are the commonly adopted approaches in various fields including medicine, econometrics and demography, among others. In the statistical literature, as indicated in Section 2, non-parametric inferences of the CORF and ORF were studied mostly under independent censoring conditions. The methods described in this paper handle the problem of informative censoring via a latent variable and thus enjoy the common advantages of latent variable models. In the proposed estimation procedures, the distributions of the censoring and latent 
Table I. The estimated expected number of events occurring every six months for HIV-negative drug users with the pointwise 95 per cent bootstrap confidence intervals.

\begin{tabular}{lcccccccc}
\hline & \multicolumn{7}{c}{ Years } \\
\cline { 2 - 8 } & $0-0.5$ & $0.5-1.0$ & $1.0-1.5$ & $1.5-2.0$ & $2.0-2.5$ & $2.5-3.0$ & $3.0-3.5$ & $3.5-4.0$ \\
\hline Number of events & 0.2359 & 0.2721 & 0.2331 & 0.3404 & 0.3136 & 0.4150 & 0.3675 & 0.3291 \\
Lower bound & 0.1778 & 0.2125 & 0.1867 & 0.2733 & 0.2470 & 0.3434 & 0.3059 & 0.2684 \\
Upper bound & 0.3069 & 0.3354 & 0.2916 & 0.4106 & 0.3859 & 0.4894 & 0.4408 & 0.3964 \\
\hline
\end{tabular}

Table II. The estimated expected number of events occurring every six months for HIV-positive drug users with the pointwise 95 per cent bootstrap confidence intervals.

\begin{tabular}{lllllllll}
\hline & \multicolumn{7}{c}{ Years } \\
\cline { 2 - 9 } & $0-0.5$ & $0.5-1.0$ & $1.0-1.5$ & $1.5-2.0$ & $2.0-2.5$ & $2.5-3.0$ & $3.0-3.5$ & $3.5-4.0$ \\
\hline Number of events & 0.3930 & 0.4625 & 0.4602 & 0.6173 & 0.6322 & 0.6467 & 0.7776 & 0.6733 \\
Lower bound & 0.3026 & 0.3568 & 0.3642 & 0.4612 & 0.4750 & 0.4561 & 0.5808 & 0.4561 \\
Upper bound & 0.4932 & 0.6004 & 0.5870 & 0.7736 & 0.8411 & 0.8190 & 0.9948 & 0.9267 \\
\hline
\end{tabular}

variables are both treated as nuisance functions and we avoid modelling and estimation of the two distributions by proper procedures. It is shown by simulation and data analyses that, in the presence of informative censoring, the proposed methods are very effective for eliminating bias.

\section{ACKNOWLEDGEMENTS}

The authors thank Drs Dave Vlahov and Steffanie Strathdee at Johns Hopkins University for providing the anonymous ALIVE data. Provision of the data was supported by NIDA grants DA04334 and DA08009. The first author's research was supported by the National Institute of Health grants R01 HD38209 and R01 MH56639.

\section{REFERENCES}

1. Vlahov D, Anthony JC, Muñov A, Margolick J, Nelson KE, Celentano DD, Solomon L, Polk BF. The ALIVE Study: a longitudinal study of HIV-1 infection in intravenous drug users: description of methods. Journal of Drug Issues 1991; 21:759-776.

2. Wang M-C, Chang S-H. Nonparametric estimation of a recurrent survival function. Journal of American Statistical Association 1999; 94:146-153.

3. Prentice RL, Williams BJ, Peterson AV. On the regression analysis of multivariate failure time data. Biometrika $1981 ; 68: 373-379$.

4. Nelson WB. Graphical analysis of system repair data. Journal of Quality Technology 1988; 20:24-35.

5. Lawless JF, Nadeau C. Some simple robust methods for the analysis of recurrent events. Technometrics 1995; 37:158-168.

6. Pepe MS, Cai J. Some graphical displays and marginal regression analyses for recurrent failure times and time dependent covariates. Journal of the American Statistical Association 1993; 88:811-820.

7. Cook RJ, Lawless JF. Marginal analysis of recurrent events and a terminating event. Statistics in Medicine 1997; 16:911-924.

8. Gasser T, Müller H-G. In Kernel Estimation of Regression Functions. Smoothing Techniques for Curve Estimation, Gasser T, Rosenblatt M (eds). Spring Lecture Notes in Mathematics No. 757, Springer-Verlag: Berlin, 1978; 23-68. 
9. Lawless JF, Nadeau C, Cook RJ. Analysis of rate and mean functions for recurrent events. Proceedings of the 1st Seattle Symposium in Biostatistics 1997; 37-39.

10. Bartoszyński R, Brown BW, McBride CM, Thompson JR. Some nonparametric techniques for estimating the intensity function of a cancer related nonstationary Poisson process. Annals of Statistics 1981; 9:1050-1060.

11. Woodroofe M. Estimating a distribution function with truncated data. Annals of Statistics 1985; 13:163-177.

12. Wang M-C, Jewell NP, Tsai W-Y. Asymptotic properties of the product limit estimate under random truncation. Annals of Statistics 1986; 14:1597-1605.

13. Wang M-C, Qin J, Chiang C-T. Analyzing recurrent event data with informative censoring. Journal of American Statistical Association 2001 (in press). 